\title{
Are Ionic Liquids Good Boundary Lubricants? A Molecular Perspective
}

\author{
Romain Lhermerout, Christophe Diederichs and Susan Perkin * \\ Department of Chemistry, Physical and Theoretical Chemistry Laboratory, University of Oxford, \\ Oxford OX1 3QZ, UK; romain.lhermerout@chem.ox.ac.uk (R.L.); christophe.diederichs@univ.ox.ac.uk (C.D.) \\ * Correspondence: susan.perkin@chem.ox.ac.uk; Tel.: +44-186-527-5496
}

Received: 18 December 2017; Accepted: 10 January 2018; Published: 16 January 2018

\begin{abstract}
The application of ionic liquids as lubricants has attracted substantial interest over the past decade and this has produced a rich literature. The aim of this review is to summarize the main findings about frictional behavior of ionic liquids in the boundary lubrication regime. We first recall why the unusual properties of ionic liquids make them very promising lubricants, and the molecular mechanisms at the origin of their lubricating behavior. We then point out the main challenges to be overcome in order to optimise ionic liquid lubricant performance for common applications. We finally discuss their use in the context of electroactive lubrication.
\end{abstract}

Keywords: ionic liquid; boundary lubrication; molecular friction; nanotribology

\section{Introduction}

Friction is a very general phenomenon that appears as soon as one tries to set two bodies in relative motion [1,2]. It is a dissipative process during which a part of the input energy originally injected to produce a global motion is in fact converted into heat. Friction therefore plays a crucial role in many applications, for which the heat production has to be maximized (matches, tyres, brakes), or minimized (turbines, motors), or at least controlled. Adding a liquid between the two solids is a well-known way to influence friction, and the lubrication effect dramatically depends on the nature of the liquid used. In general, real surfaces are rough so friction takes place on many asperities (Figure 1a). The link between the lubrication mechanisms on a single asperity and the macroscopic motion is complex, because of the possible emergence of collective effects. In the following, we will focus on the so-called boundary lubrication regime, namely when the two solids are separated by a liquid film of a few molecules thickness on a single asperity. For reviews about macroscopic friction across ionic liquids, we refer to [3-5].

A "good lubricant" could be defined in terms of three principal requirements. (i) The friction coefficient achieved should be controlled. A very low friction, or superlubricity is not necessarily required in applications; instead it is often desirable to have highly controlled friction in order to create very reproducible motion in response to an external force or perturbation. Examples are the MEMS gyroscopes and capacitive accelerometers found in typical mobile phones/devices; (ii) The liquid film should be able to sustain the relevant pressure without being squeezed-out. Typical pressure experienced by boundary films on rough surfaces can indeed be extremely high because the normal load is concentrated on microscopic asperities. The sustained presence of liquid not only maintains lubrication but often also serves to reduce wear; (iii) The lubrication performance should be robust, i.e., minimally dependent on changes in the environment. This last condition is particularly challenging given the multiple perturbations potentially at play (high temperatures, pressures, shear stresses, contamination by moisture, etc.) that can lead to many uncontrolled modifications of the system by complex tribological processes (wear, phase transition, (electro)chemical decomposition, etc.). 
(a)

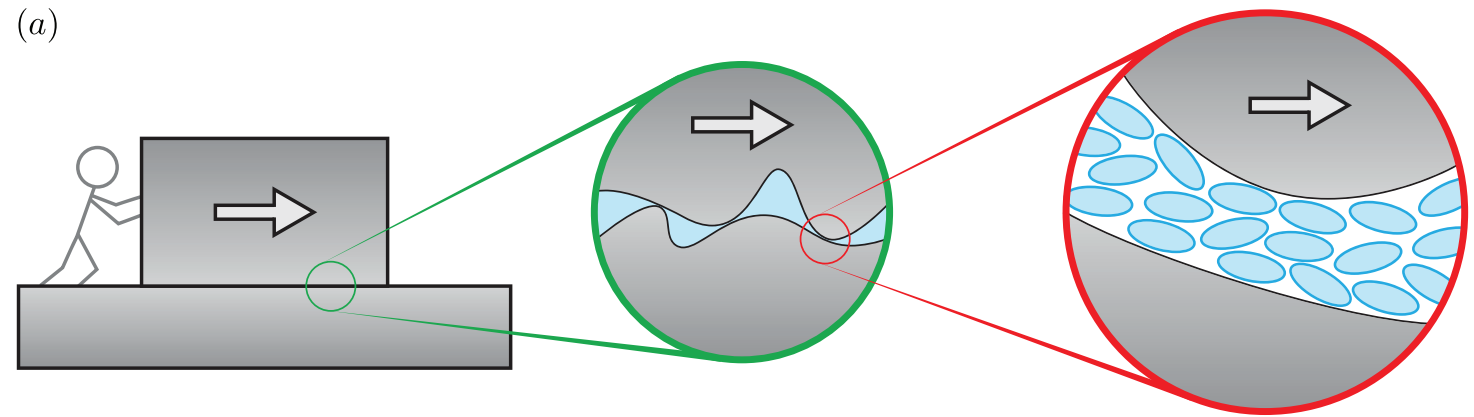

$(b)$

$F_{\mathrm{L}}$

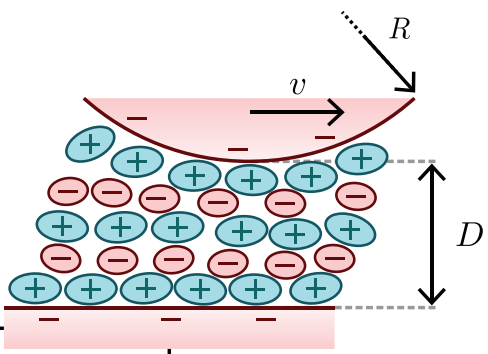

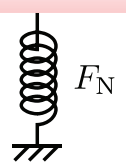

$(c)$
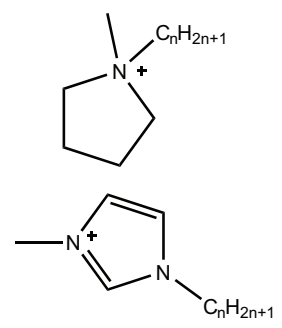

$R_{4} / /$ $(d)$<smiles>CS(=O)(=O)[N-]S(=O)(=O)[18F]</smiles><smiles>CCOS(=O)(=O)[O-]</smiles>

|

Figure 1. (a) Friction is a multi-scale phenomenon: the macroscopic sliding of usual surfaces is slowed down by dissipation involving many mesoscopic asperities, where the lubricant should act at the molecular scale; (b) Schematic and relevant quantities of a typical measurement of friction on a single contact across an ionic liquid; (c) Examples of cations common in ionic liquids, from top to bottom: 1-alkyl-1-methylpyrrolidinium $\left[\mathrm{C}_{n} \mathrm{C}_{1} \mathrm{Pyrr}\right]^{+}$, 1-alkyl-3-methylimidazolium $\left[\mathrm{C}_{\mathrm{n}} \mathrm{C}_{1} \mathrm{Im}\right]^{+}$, phosphonium $\left[\mathrm{R}_{\mathrm{i}} \mathrm{P}\right]^{+}$; (d) Examples of anions common in ionic liquids, from top to bottom: bis[(trifluoromethane)sulfonyl]imide $\left[\mathrm{NTf}_{2}\right]^{-}$(also commonly known as TFSI), ethylsulfate $\left[\mathrm{EtSO}_{4}\right]^{-}$, tris(pentafluoroethyl)trifluorophosphate $[\mathrm{FAP}]^{-}$.

Ionic liquids are pure salts that are liquid under room temperature-pressure conditions. They exhibit an exceptional stability (low volatility, nonflammability, wide electrochemical and thermal windows), which makes them very promising systems for lubrication and many other applications (energy storage, catalysis, electrowetting, etc.) [6-8]. Additionally, it has been observed that ionic liquids are very hard to squeeze-out and can produce low friction [9], irrespective of the temperature [10], thus they could potentially be very good lubricants.

In this review, we will summarize the main questions, findings and perspectives in the field of friction across ionic liquids in the boundary lubrication regime. The aims are to discuss the current state of knowledge regarding the molecular-level mechanisms of dissipation across ionic liquid films, and, from this, to identify important molecular considerations when designing ionic liquid lubricants for various purposes. The paper is organized as follows. In Section 2, we will review studies giving insight into the relation between the molecular structure and the frictional behavior. In Section 3, we will focus on how the lubrication performances vary if the ionic liquid is not pure, i.e., with a small fraction of water present or diluted in a solvent. Section 4 will be dedicated to techniques to "switch" friction properties, namely to change friction by varying external parameters and in a reversible way.

\section{The Molecular Mechanisms of Friction across Ionic Liquids}

The Surface Force Balance/Apparatus and the Colloidal Probe Atomic Force Microscope are two commonly used techniques to directly measure friction in the boundary lubrication regime [11]. The principle of these experiment is sketched in Figure $1 \mathrm{~b}$. The two solid surfaces are at the same time curved at large scale (radius of curvature $R \sim 1 \mathrm{~cm}$ in typical Surface Force Balance experiments) and 
smooth at the molecular scale, to ensure a single contact point. The liquid thickness $D$ is set to a known value with a precision better than the molecular size, one surface is moved laterally at a velocity $v$ and the corresponding normal force $F_{\mathrm{N}}$ and lateral force $F_{\mathrm{L}}$ are measured.

When confined between two solid surfaces at the nanoscale, an ionic liquid tends to be squeezed-out discontinuously, by discrete steps, arising from the organized arrangement of molecules in ordered layers. This produces a damped oscillation for the force profile, called structural or solvation force (Figure 2a). Such phenomena are not specific to ionic liquids; structural forces are observed with apolar solvents, salt solutions, polymer melts, etc. [12-15]. However, ionic liquid structure consists of alternating layers of excess cation density and excess anion density, and one squeeze-out event corresponds in fact to the squeeze-out of an electroneutral "slab" which can be considered, approximately, as two layers (one cation- and one anion-excess) together [9,16-22]. In several cases of long-chain and geometrically irregular ionic liquids the squeeze-out behaviour is found to become continuous, rather than discontinuous. The amplitude of the oscillations are in general higher for ionic liquids, arising from the high electrostatic barriers to reorganisation, and the last layer of cations is usually never squeezed-out, even under normal pressures of $\sim 10 \mathrm{MPa}$. The condition (ii) to have a good lubricant, i.e., the ability to resist squeeze-out is then very well fulfilled by ionic liquids.

$(a)$

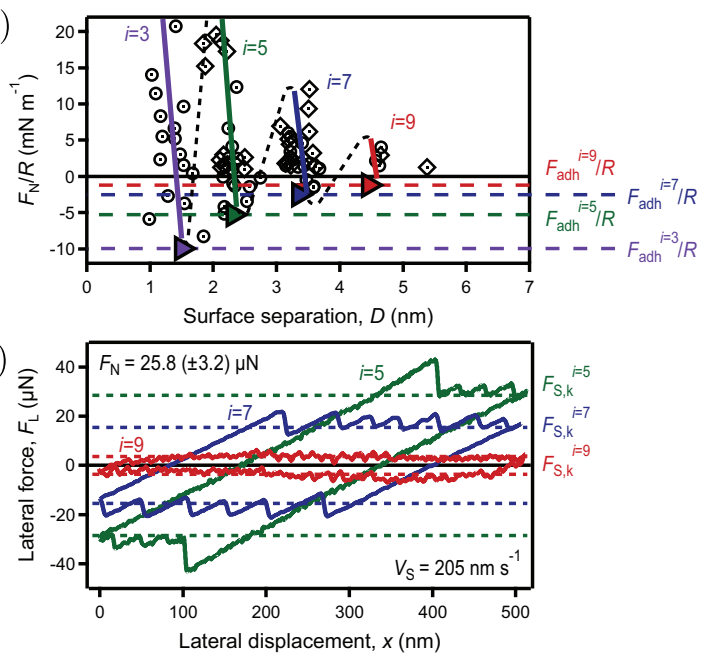

(d)

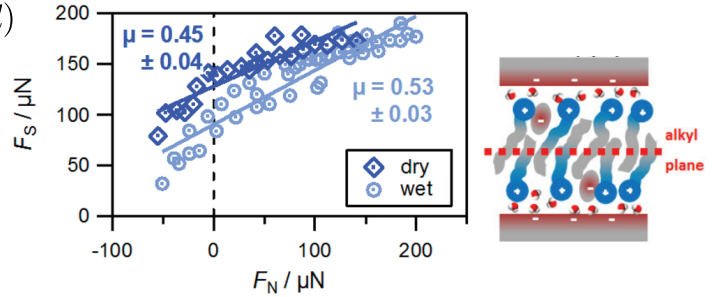

$(c)$

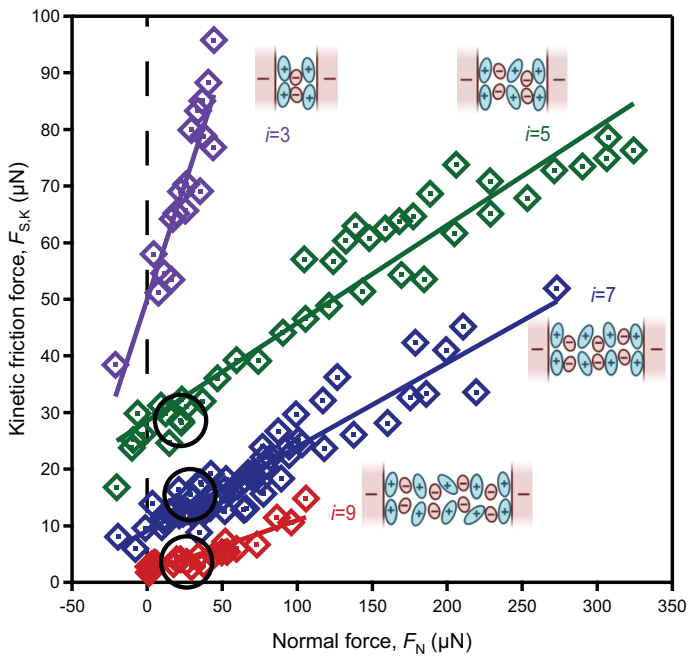

$(e)$

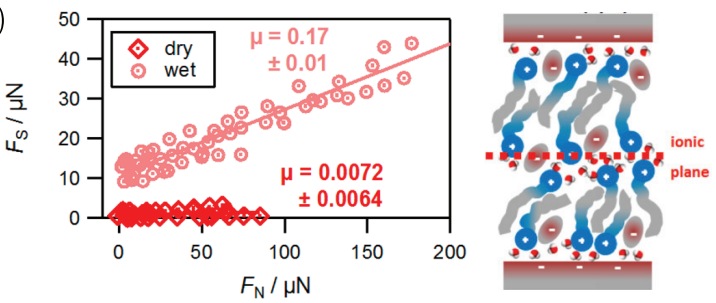

Figure 2. (a-c) Forces between mica surfaces across a structured nanofilm of $\left[\mathrm{C}_{4} \mathrm{C}_{1} \mathrm{Pyrr}\right]\left[\mathrm{NTf}_{2}\right]$, measured with a Surface Force Balance: (a) oscillatory profile of the normal force rescaled by the radius of curvature of the single-asperity contact (proportional to the interaction energy between parallel plates of the same material at the separation of closest approach, according to the Derjaguin approximation); (b) stick-slip evolution of the lateral force as a function of the lateral displacement, for three different numbers of layers at same load and velocity, indicated by the black circles in (c); (c) kinetic friction force/normal force relationship, showing quantized friction. Figures adapted from [23]—Published by the Physical Chemistry Chemical Physics Owner Societies; (d,e) Similar friction measurements, performed with (d) one or (e) two bilayers of $\left[\mathrm{C}_{10} \mathrm{C}_{1} \mathrm{Pyrr}\right]\left[\mathrm{NTf}_{2}\right]$, under dry or wet conditions as indicated in the legends. Water molecules are used as "tracers" to deduce the sliding planes, indicated in the cartoons. Adapted with permission from [24] - Copyright 2014 American Chemical Society. 
The characteristic features of the frictional response across ionic liquids are qualitatively similar to that observed with apolar liquids. A stick-slip behavior is obtained under certain conditions of velocity and load, which is typical of solid-like friction (Figure 2b). The mechanism of molecular reorganisation during the 'slip' phases across apolar liquids has been the matter of intense discussion for many years: the principal distinction being between successive freezing-melting transitions in the nanofilm, or inter-layer (or wall) slip [25-36]. It has been argued that, in the case of ionic liquids, slip (or 2D in-plane melt) is likely to be more favourable than full-film (3D) melting during the slip phase [23,24].

Several papers report "quantized friction" when measuring the friction force as a function of the load and of the number of layers in the film $[9,23,37,38]$. The quantized description refers to the discrete multi-valued nature of the friction force corresponding to each single value of applied load; this phenomenon is not unique to ionic liquids and will arise in every case of an oscillatory normal force law. As we clearly see in Figure 2c, each liquid composition corresponds to a different linear friction-load relationship. The variation of the intersect can be understood as the adhesion contribution, that increases when the number of layers in the film decreases, and is also a feature shared with apolar molecules $[39,40]$.

There are, however, two major differences between the frictional responses of ionic liquids and apolar liquids. First, with ionic liquids the friction coefficients (i.e., slope in the friction/load law) is also quantized and increases monotonically when the number of layers decreases, while for apolar liquids variation of friction coefficient with number of layers is barely measurable. Secondly, friction coefficients values can be much lower for ionic liquids than for apolar molecules. For example, it goes from $\sim 0.1$ to $\sim 0.8$ for $\left[\mathrm{C}_{4} \mathrm{C}_{1}\right.$ Pyrr] $\left[\mathrm{NTf}_{2}\right]$ [23], from $\sim 0.007$ to $\sim 0.5$ for $\left[\mathrm{C}_{10} \mathrm{C}_{1} \mathrm{Pyrr}\right]\left[\mathrm{NTf}_{2}\right]$ [24], while it is $\sim 1.1$ for octamethylcyclotetrasiloxan (OMCTS) [9] (all between mica in SFB). This relatively low resistance to shear (condition (i)) also makes ionic liquids promising lubricants.

Why is the frictional response of ionic liquids different to that of apolar liquids? It is well established that the ordered layers in a confined film of ionic liquid are distinctly different to that of an apolar liquid, with oscillations in the charge density away from the surface corresponding to alternating layers of excess cation-density and excess anion-density and strongly dependent on the surface polarity and charge [7]. Successive layers away from the boundary walls can have differing composition-ratio of cations to anions - in contrast to the uniform composition in a pure apolar liquid. The influence of this structure on the lubrication properties was illuminated by comparisons made between measurements with dry and wet ionic liquids. Figure 2d,e shows measurements made with $\left[\mathrm{C}_{10} \mathrm{C}_{1}\right.$ Pyrr $]\left[\mathrm{NTf}_{2}\right]$, which is organized in bilayers [22,24], in dry or wet conditions. A key finding of this study is that the friction coefficient for a single bilayer is independent of the water content, whereas it is enhanced by more than an order of magnitude for two bilayers when the liquid is wet. If one makes the reasonable assumption that the water molecules preferentially sits close to the charged heads of the cations, we can deduce that the sliding plane is on the alkyl chains for the single bilayer, while it is on the middle ionic region for the two bilayers. Because of the strong binding of the charged groups on the charged surface, and the variation in friction coefficient with film thickness (number of bilayers present), interlayer slip is expected to be more likely than wall slip. Depending on the number of layers separating the solid, new possible sliding planes appear with different compositions (alkyl chains or charged groups), leading to different resistance to sliding.

Even in the boundary lubrication regime, where the solid surfaces are separated by a liquid film of only a few molecular diameters thickness, one should keep in mind that friction processes usually involve macroscopic dimensions in the plane of the contact. Friction thus results from the collective response of the whole film, and this has been stressed by the observation of "microslip" events, i.e., temporary reduction in friction, that can sometimes become avalanches, i.e., collective slip [41] (Figure $3 b, c)$. It is notable that such collective phenomena are determined by molecular features and in particular the structure and dynamics of the most highly confined regions of a macroscopic contact. In considering this interesting matter, we note that a similar scenario arises with the (normal) 
oscillatory structural force. In that case the oft-posed question is: even if the molecules are locally ordered, a geometry involving curved surfaces (e.g., crossed cylinders, or sphere on flat) means that the liquid film thickness increases continuously with distance away from the point of closest approach. At one location the film thickness may correspond to an integer number of molecular diameters, while at another point this is not the case. So why do we see an oscillating force? As was addressed long ago in the early measurements of such forces, the (perhaps counterintuitive) result of integrating all the forces across the contact returns an oscillatory function with parameters identical to that of plane-parallel approach with a phase shift [12]. Heuristically, we can understand that the contribution of the thinnest film is most substantial. It is clear that understanding the emergence of collective slip and the precise features of the stick-slip pattern is the key to quantitatively bridge the molecular scale to the observed frictional behavior.

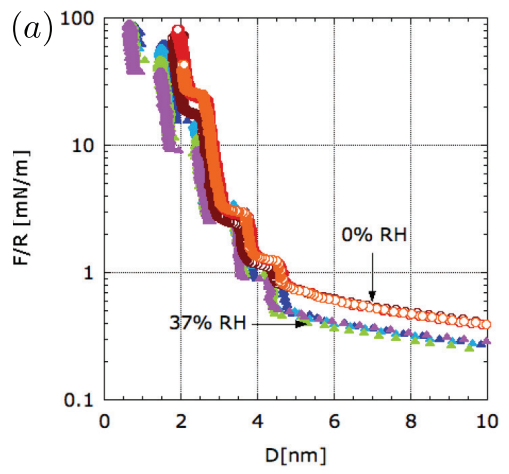

(d)

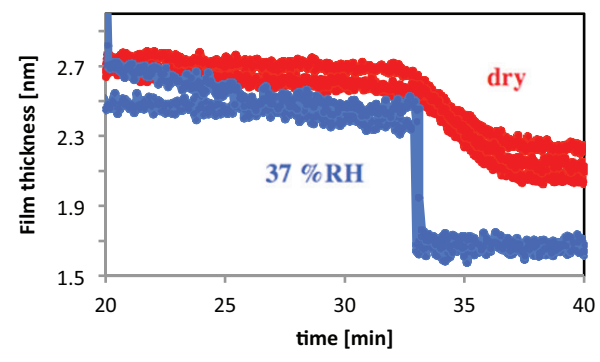

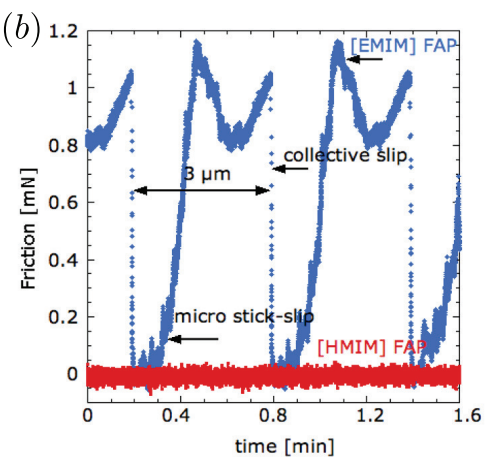

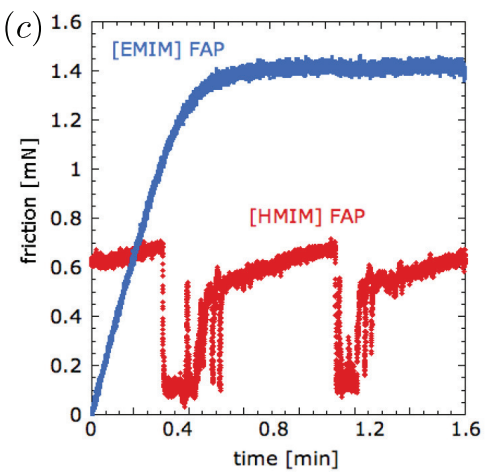

(e)

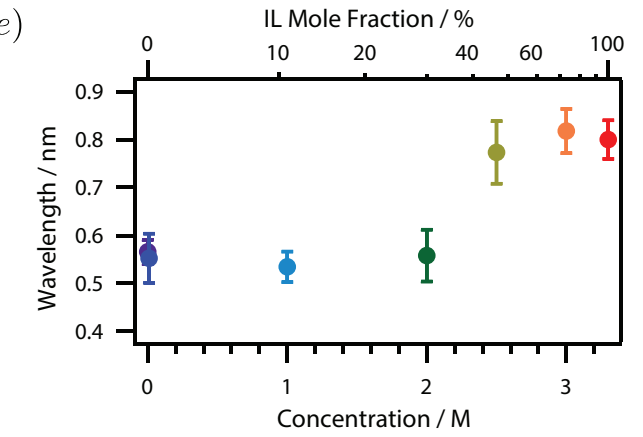

Figure 3. (a-d) Forces between mica surfaces across structured nanofilms of $\left[\mathrm{C}_{2} \mathrm{C}_{1} \mathrm{Im}\right][\mathrm{FAP}]$ and $\left[\mathrm{C}_{6} \mathrm{C}_{1} \mathrm{Im}\right][\mathrm{FAP}]$, measured with a Surface Force Apparatus: (a) profile of the normal force rescaled by the radius of curvature of the single-asperity contact; (b) temporal evolution of the friction force in dry condition; (c) temporal evolution of the friction force in wet condition; (d) squeeze-out dynamics. Reprinted with permission from [41]-Copyright 2014 American Chemical Society; (e) Mean thickness of the structured layers for different mixtures of $\left[\mathrm{C}_{4} \mathrm{C}_{1} \mathrm{Pyrr}\right]\left[\mathrm{NTf}_{2}\right]$ in propylene carbonate. Reprinted with permission from [42]—Copyright 2017 by the American Physical Society.

Given the strong influence of molecular structure and orientation on the friction and dissipation across molecular films, it is clear that altering the molecular architecture and chemistry is a route to tune friction. For example, it has been shown that structural and friction forces vary when using ion pairs of different size and flexibility (alkyl chain length) or symmetry (bilayers structure), or different interactions [43-47] (see commonly used ions in Figure 1). The range of ionic liquids available commercially has grown substantially over the recent few years; many systems still have to be explored in order to discover the extent to which behavior is universal or, on the contrary, to discover new properties and possibilities.

So far, the role of surface chemistry has been investigated only a little and is far from being fully understood. This is mainly because the criterion for surfaces to possess atomic-scale smoothness precludes the possibility of systematically measuring behaviour at single asperity contacts for a 
wide range of materials. However, recent studies have demonstrated how ionic liquid structure and dynamics may vary significantly according to the confining surfaces. For example, oscillatory normal force profiles for $\mathrm{Au}(100)$ have been found to be less pronounced than for mica, while at comparable surface potentials [48]. Nanoscopic friction response can also depends greatly on surface specific molecular anchoring effects [47]. As detailed in Section 4, surface chemistry becomes increasingly important when investigating potential or field dependent effects.

\section{Small Fractions of Molecular Liquid Can Dramatically Influence Lubrication of Ionic Liquids}

It has long been known that physical properties (e.g., viscosity, conductivity) of ionic liquids are dramatically altered by the presence of small amounts of water, and that removal all water is challenging. Ideal model experiments can be performed under rather dry conditions (typically $\ll 100$ ppm water for the less hydrophilic ionic liquids), achieved by drying the liquid under vacuum and elevated temperature prior to experiments which are then performed in a closed chamber containing a desiccant and purged with a dry gas. However the hygroscopic nature of most ionic liquids and the ambient humidity involved in most application environments mean that it becomes important to understand the influence of water and other molecular components in ionic liquids. Solid surfaces are generally charged, and a small water concentration in the bulk can indeed lead to high interfacial concentrations under confinement [49].

Several studies report systematic measurements by varying the water concentration. Regarding the normal force, it has been shown that the structural force profile is significantly modified. An increase of the layer thicknesses is observed for some liquids, which is intuitively rationalized by the intercalation of water molecules, or "swelling" of the ions (Figure 3d) [24,41,50]. More intriguing is the thinning of the layers for other liquids, due to subtle structural changes like molecule reorientations or composition changes $[41,49,50]$. The extension of the structural force region and the amplitude of adhesive minima can even be enhanced, interpreted as the ability of water to increase surface charge [51]. Several studies reported a reduction of the maxima, meaning that water facilitates the squeeze-out of the ionic liquid (Figure 3a) $[41,49,50]$.

The presence of water also affects the dynamics within the layers $[41,49,50]$. The squeeze-out transition has been found to be much faster with water (Figure 3d). However water also tends to significantly increase friction; perhaps a counterintuitive result. Experiments with two bilayers of $\left[\mathrm{C}_{10} \mathrm{C}_{1}\right.$ Pyrr $]\left[\mathrm{NTf}_{2}\right]$ show an increase of the friction coefficient by more than an order of magnitude when water is present within the layers [24] (Figure 2e). Similarly, friction response of dry $\left[\mathrm{C}_{6} \mathrm{C}_{1} \operatorname{Im}\right][\mathrm{FAP}]$ has been found below instrumental sensitivity, whereas friction is substantial in wet conditions [41] (Figure 3b,c). This trend has been confirmed by fluorescence anisotropy experiments [52] and by simulations [49]. Humidity can even change the shape of the friction response: adding water in $\left[\mathrm{C}_{2} \mathrm{C}_{1}\right.$ Pyrr $]\left[\mathrm{NTf}_{2}\right]$ can induce a transition from stick-slip behavior to continuous sliding (Figure 3b,c).

Such substantial effects of water on the lubricating performance of ionic liquids may at first sight appear problematic for applications, as moisture is present almost everywhere and lubricated regions usually cannot be perfectly sealed. However it is important to note that the majority of the above molecular-resolution studies were performed with highly hydrophobic, i.e., water-immiscible (though nonetheless hygroscopic) ionic liquids-motivated by the need to approach the water-free limit under laboratory conditions-and therefore the presence of water in these fluids is likely to be more substantial than for more hydrophilic (water-miscible) ionic liquids. It will be important to compare in future the effect of water on hydrophilic vs. hydrophobic ionic liquids. Relatedly, ions containing corrosive and toxic halogens are problematic for applications, and there is a real need for "green" lubricants $[53,54]$. Designing ionic liquids which are at the same robust to the inclusion of small amounts of water from the environment, environmentally friendly and with desired lubrication ability is a present and important challenge.

Another strategy to tackle this difficulty is to use ionic liquids as additives in solutions, such that the mixture is not sensitive to ambient humidity. This approach is also motivated by the high current cost of ionic liquids compared to usual lubricants and oils. The main challenge is then: could it be 
possible to achieve in solution lubricating performances which are comparable to those in pure state? In this respect, measurements of the structural force for mixtures of $\left[\mathrm{C}_{4} \mathrm{C}_{1} \mathrm{Pyrr}\right]\left[\mathrm{NTf}_{2}\right]$ and propylene carbonate seem promising [42]. While the concentration of ionic liquid has been varied continuously, the thickness of the layers abruptly change from the pure propylene carbonate behavior to the pure ionic liquid behavior at a threshold mole fraction of $\sim 40 \%$ (Figure $3 \mathrm{e}$ ). There is, therefore, a wide range of concentrations for which the pure ionic liquid structure is preserved, and one can expect a similar behavior for the frictional response. Propylene carbonate is not a realistic molecular solvent for lubricant applications because of its volatility, instead ionic liquid/oil mixtures would be more suitable candidate mixtures. It is important to note, also, that the polarity and permittivity of the polar vs. nonpolar solvents is likely to impact the structures formed. Studies in this direction showed that, if the compounds are well chosen, the ions can maintain a boundary layer and so preserve good lubricant properties; this is likely to be aided by the surface activity of the ions which leads to a higher concentration at the interface than in the bulk [55,56]. One could ask: what is the advantage of ionic liquid additives, compared to the already-used surfactant additives? The main difference is that in their pure form under ambient conditions ionic liquids are liquid, by definition, while surfactants are generally solid. Surfactants form aggregates, or even (liquid) crystal phases, at sufficiently high concentration, therefore as solvent fraction decreases (typically by evaporation or oxidation of solvent) the surfactant content can increase, eventually to a problematic level. Such problems are avoided for ionic liquids, which perform effectively at high concentrations. Dealing with mixtures remains challenging in general and unexpected behaviors can emerge [57,58]; a wide and careful survey of mixtures of ionic liquids with low-volatility molecular solvents and the resulting interfacial properties will be essential for progress in this direction.

\section{Ionic Liquids Can Be Manipulated to Externally Switch Friction}

The previous sections focused on "passive" lubrication, i.e., systems for which the lubrication properties are determined by the choice of chemical compounds (lubricant, surface). There is also a strong interest for "smart" lubricants, whose properties could be switched on demand. An external and reversible control of friction would be very useful for applications like micro/nanoelectromechanical systems (MEMS/NEMS), where the production or the stopping of a motion could be performed more efficiently by changing the friction coefficient in addition or instead of the load. Ionic liquids are very promising systems for this purpose, because charges in general can be manipulated by external electric stimuli (electroactive lubrication), and ionic liquid in particular have wide electro-chemical windows. Achieving such external control is also an opportunity to build a better understanding of the molecular mechanisms of friction.

Important efforts have been made to interact with the ionic liquid by changing the surface charge. Numerical simulations showed that modifying the surface charge can affect the liquid composition and its structure, and also the frictional response [59-61]. Figure 4b,c shows that the friction-load relationship depends on the surface charge, but that this evolution is not monotonic. At low surface charges, most of the sliding occurs at the liquid/solid interface, and dissipation increases while the first layer of counter-ions on each electrode is more on more coupled to the wall. At high surface charges, this first layer is so rigidly coupled to the solid that inter-layer slip becomes dominant, and this can ultimately lead to superlubricity.

Usually, boundary lubrication experiments are performed with dielectric surfaces (mica, silica, sapphire etc.), for which the surface charge is not controlled. It depends on the material, the $\mathrm{pH}$ of the solution, and complex specific interactions between at the solid/liquid interface. For instance, mica at no applied potential has a significant negative surface charge due to desorption of surface $\mathrm{K}^{+}$ions into solution. Surface charge control is generally reached by using one or two conductive surfaces (gold, graphite, etc.). Doing so, the control parameter is in fact not the surface charge, but the surface potential. Many experiments in this field have been performed by Colloidal Probe Atomic Force Microscope, because of the difficulty to produce conductive electrodes that are clean and smooth 
at the molecular scales at the scale of the probe. It has been shown that controlling the surface potential is a way to change the boundary film composition, as revealed by the change of the structural force profile [20]. Figure $4 a$ shows friction measurements for $\left[\mathrm{C}_{4} \mathrm{C}_{1} \mathrm{Pyrr}\right][\mathrm{FAP}]$ confined between silica and a gold electrode of controlled potential [62]. When a negative potential is applied to the gold electrode, the liquid film is enriched in cations, which produces a lower friction coefficient, presumed to be due to the relatively good lubricating alkyl chains of the cation. The sign of the effect depends on the relative lubricity of the two ions [63], and the effect is not necessarily monotonic, because of specific interactions or subtle reorientations mechanisms. For example, highly ordered pyrolytic graphite (HOPG) surfaces have strong solvophobic interactions with non-polar chains of substrate molecules [64], greatly changing friction response to surface potential change when compared with Gold surfaces $[65,66]$. For a particular choice of the liquid and surface chemistry, it has even been shown that it is possible to switch superlubricity [65]. In general, these studies at controlled surface charge are powerful tools to try to link the liquid structure to the frictional behavior and to identify the dissipation processes at play.

(a)

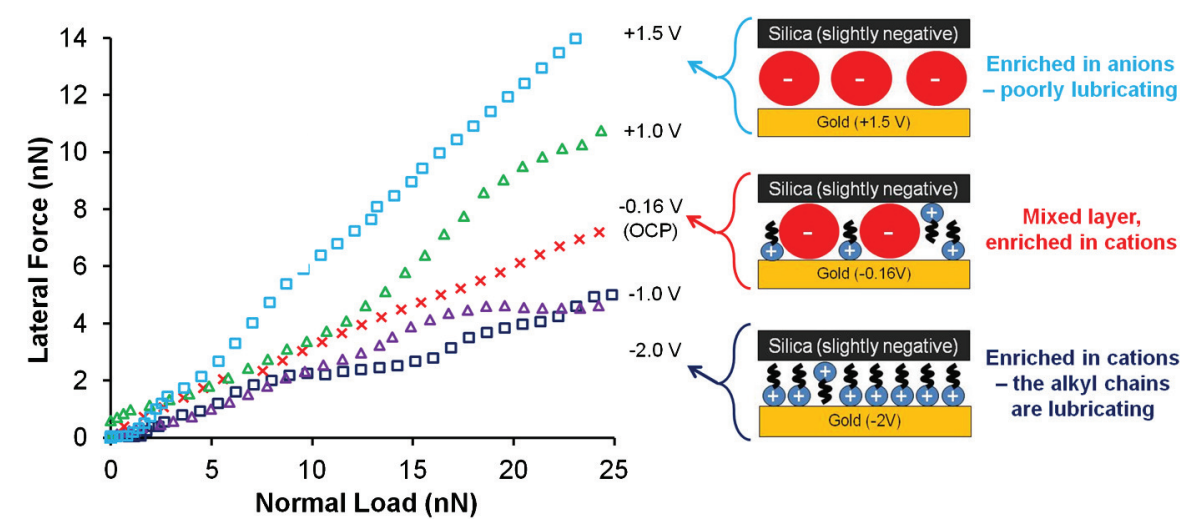

(b)

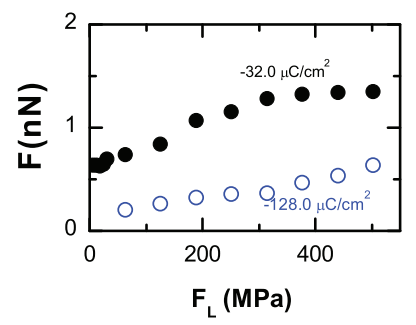

$(c)$

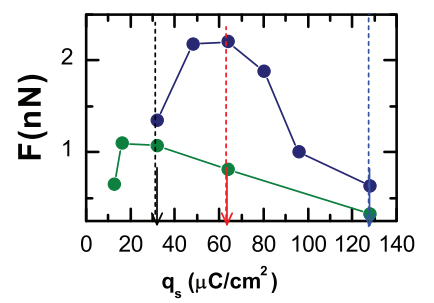

$(d)$

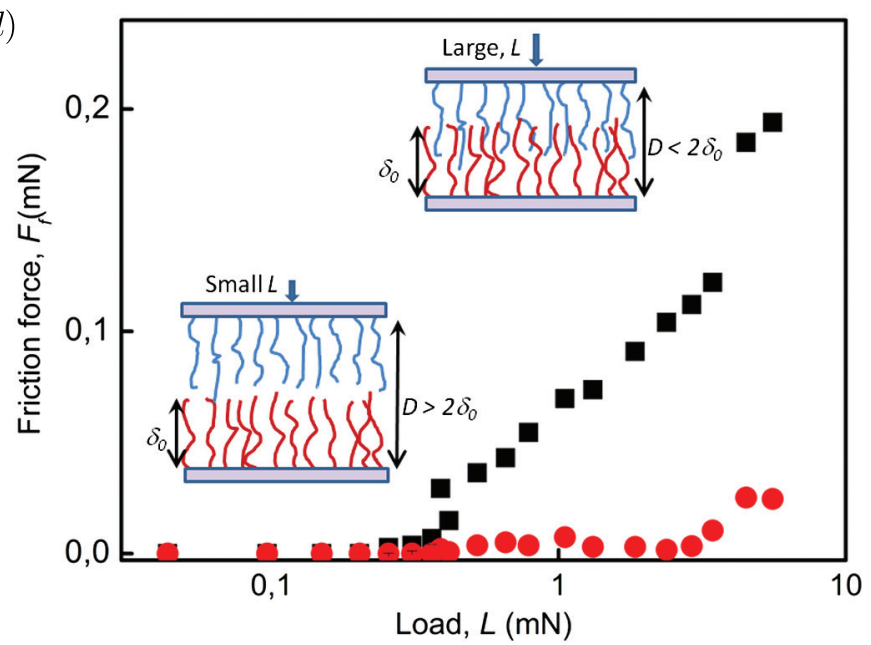

Figure 4. (a) Friction force/normal force relationship for $\left[\mathrm{C}_{4} \mathrm{C}_{1} \mathrm{Pyrr}\right][\mathrm{FAP}]$ between silica and a gold electrode at different potentials, measured with a Colloidal Probe Atomic Force Microscope. Reprinted with permission from [62] - Copyright 2012 by the American Physical Society; (b,c) Dependence of the friction force on the load and the electric charge of the confining surfaces obtained numerically: (b) friction force/normal force relationship for two surface charges; (c) friction force/surface charge relationship for two loads (188 MPa in green and $500 \mathrm{MPa}$ in blue). Reproduced with permission from [59]; (d) Friction force/normal force relationship for polyelectrolyte brushes coating mica in a Surface Force Apparatus, with (red circles) and without (black squares) an applied alternating electric field. Reprinted with permission from [67]—Copyright 2012 by the American Physical Society. 
Another strategy, not explored yet to our knowledge, would be to apply an electric field across the nano-film. Such control can be achieved with a Surface Force Apparatus, for which the metallic mirrors at the back of the mica can be used as electrodes. This method has been used for polyelectrolyte coatings immersed in water and for liquid crystals [67-69]. In Figure $4 \mathrm{~d}$ is reproduced the friction-load characteristics between to mica surfaces bearing polyelectrolyte brushes. When an alternating electric field is applied, friction is dramatically reduced because interpenetration of the polymer chains is prevented. Similarly, one can expect for ionic liquids that the electric field induces structural changes, and so modifies the lubrication properties of the film.

\section{Conclusions}

In this review, we gather the main findings about frictional behavior of ionic liquids in the boundary lubrication regime. Ionic liquids are promising lubricants because of their ability to form structured boundary layers which are strongly bound on charged or polar surfaces, promoting a low-dissipating inter-layer slip. The main limitations are their sensitivity to ambient moisture, their possible toxicity and their cost, that could be overcame by developing green ionic liquid additives. Their strong and switchable response to external electrical stimuli make them systems of choice for smart applications [70]. Interesting properties or even new behaviors could emerge from zwiterrionic ionic liquids [71], which have to our knowledge not yet been investigated.

Acknowledgments: S.P. and R.L. are supported The Leverhulme Trust (RPG-2015-328) and the ERC (under Starting Grant No. 676861, LIQUISWITCH). R.L. is supported by the EPA Cephalosporin Junior Research Fellowship and Linacre College (University of Oxford). S.P. is grateful for research leave enabled by the Philip Leverhulme Prize.

Conflicts of Interest: The authors declare no conflict of interest.

\section{References}

1. Bowden, F.P.; Tabor, D. The Friction and Lubrication of Solids; Oxford University Press: Oxford, UK, 1950.

2. Kardar, M.; Golestanian, R. The "friction" of vacuum, and other fluctuation-induced forces. Rev. Mod. Phys. 1999, 71, 1233-1245.

3. Bermúdez, M.D.; Jiménez, A.E.; Sanes, J.; Carrión, F.J. Ionic Liquids as Advanced Lubricant Fluids. Molecules 2009, 14, 2888-2908.

4. Minami, I. Ionic Liquids in Tribology. Molecules 2009, 14, 2286-2305.

5. Palacio, M.; Bhushan, B. A Review of Ionic Liquids for Green Molecular Lubrication in Nanotechnology. Tribol. Lett. 2010, 40, 247-268.

6. Hallett, J.P.; Welton, T. Room-Temperature Ionic Liquids: Solvents for Synthesis and Catalysis. Chem. Rev. 2011, 111, 3508-3576.

7. Fedorov, M.V.; Kornyshev, A.A. Ionic Liquids at Electrified Interfaces. Chem. Rev. 2014, 114, 2978-3036.

8. Hayes, R.; Warr, G.G.; Atkin, R. Structure and Nanostructure in Ionic Liquids. Chem. Rev. 2015, 115, 6357-6426.

9. Perkin, S.; Albrecht, T.; Klein, J. Layering and shear properties of an ionic liquid, 1-ethyl-3-methylimidazolium ethylsulfate, confined to nano-films between mica surfaces. Phys. Chem. Chem. Phys. 2010, 12, 1243-1247.

10. Hjalmarsson, N.; Atkin, R.; Rutland, M.W. Is the boundary layer of an ionic liquid equally lubricating at higher temperature? Phys. Chem. Chem. Phys. 2016, 18, 9232-9239.

11. Israelachvili, J.N. Intermolecular and Surface Forces; Academic Press: Cambridge, MA, USA, 2011.

12. Horn, R.G.; Israelachvili, J.N. Direct measurement of structural forces between two surfaces in a nonpolar liquid. J. Chem. Phys. 1981, 75, 1400-1411.

13. Christenson, H.K. Experimental measurements of solvation forces in nonpolar liquids. J. Chem. Phys. 1983, 78, 6906-6913.

14. Israelachvili, J.N.; Pashley, R.M. Molecular layering of water at surfaces and origin of repulsive hydration forces. Nature 1983, 306, 249-250.

15. Horn, R.G.; Israelachvili, J.N. Molecular organization and viscosity of a thin film of molten polymer between two surfaces as probed by force measurements. Macromolecules 1988, 21, 2836-2841. 
16. Horn, R.G.; Evans, D.F.; Ninham, B.W. Double-layer and solvation forces measured in a molten salt and its mixtures with water. J. Phys. Chem. 1988, 92, 3531-3537.

17. Atkin, R.; Warr, G.G. Structure in Confined Room-Temperature Ionic Liquids. J. Phys. Chem. C 2007, 111, 5162-5168.

18. Bou-Malham, I.; Bureau, L. Nanoconfined ionic liquids: Effect of surface charges on flow and molecular layering. Soft Matter 2010, 6, 4062-4065.

19. Ueno, K.; Kasuya, M.; Watanabe, M.; Mizukami, M.; Kurihara, K. Resonance shear measurement of nanoconfined ionic liquids. Phys. Chem. Chem. Phys. 2010, 12, 4066-4071.

20. Hayes, R.; Borisenko, N.; Tam, M.K.; Howlett, P.C.; Endres, F.; Atkin, R. Double Layer Structure of Ionic Liquids at the $\mathrm{Au}(111)$ Electrode Interface: An Atomic Force Microscopy Investigation. J. Phys. Chem. C 2011, 115, 6855-6863.

21. Perkin, S.; Crowhurst, L.; Niedermeyer, H.; Welton, T.; Smith, A.M.; Gosvami, N.N. Self-assembly in the electrical double layer of ionic liquids. Chem. Commun. 2011, 47, 6572-6574.

22. Smith, A.M.; Lovelock, K.R.J.; Gosvami, N.N.; Licence, P.; Dolan, A.; Welton, T.; Perkin, S. Monolayer to Bilayer Structural Transition in Confined Pyrrolidinium-Based Ionic Liquids. J. Phys. Chem. Lett. 2013, 4, 378-382.

23. Smith, A.M.; Lovelock, K.R.J.; Gosvami, N.N.; Welton, T.; Perkin, S. Quantized friction across ionic liquid thin films. Phys. Chem. Chem. Phys. 2013, 15, 15317-15320.

24. Smith, A.M.; Parkes, M.A.; Perkin, S. Molecular Friction Mechanisms Across Nanofilms of a Bilayer-Forming Ionic Liquid. J. Phys. Chem. Lett. 2014, 5, 4032-4037.

25. Israelachvili, J.N.; Kott, S.J. Shear Properties and Structure of Simple Liquids in Molecularly Thin Films: The Transition from Bulk (Continuum) to Molecular Behavior with Decreasing Film Thickness. J. Colloid Interface Sci. 1989, 129, 461-467.

26. Thompson, P.A.; Robbins, M.O. Origin of Stick-Slip Motion in Boundary Lubrication. Science 1990, 250, 792-794.

27. Klein, J.; Kumacheva, E. Confinement-Induced Phase Transitions in Simple Liquids. Science 1995, 269, 816-819.

28. Klein, J.; Kumacheva, E. Simple liquids confined to molecularly thin layers. I. Confinement-induced liquid-to-solid phase transitions. J. Chem. Phys. 1998, 108, 6996-7009.

29. Alba-Simionesco, C.; Coasne, B.; Dosseh, G.; Dudziak, G.; Gubbins, K.E.; Radhakrishnan, R.; Sliwinska-Bartkowiak, M. Effects of confinement on freezing and melting. J. Phys. Condens. Matter 2006, 18, R15, doi:10.1088/0953-8984/18/6/R01.

30. Klein, J. Frictional Dissipation in Stick-Slip Sliding. Phys. Rev. Lett. 2007, 98, doi:10.1103/PhysRevLett.98.056101.

31. Docherty, H.; Cummings, P.T. Direct evidence for fluid-solid transition of nanoconfined fluids. Soft Matter 2010, 6, 1640-1643.

32. Lei, Y.; Leng, Y. Stick-Slip Friction and Energy Dissipation in Boundary Lubrication. Phys. Rev. Lett. 2011, 107, 147801, doi:10.1103/PhysRevLett.107.147801.

33. Rosenhek-Goldian, I.; Kampf, N.; Yeredor, A.; Klein, J. On the question of whether lubricants fluidize in stick-slip friction. Proc. Natl. Acad. Sci. USA 2015, 112, 7117-7122.

34. Jee, A.; Lou, K.; Granick, S. Scrutinizing evidence of no dilatancy upon stick-slip of confined fluids. Proc. Natl. Acad. Sci. USA 2015, 112, 11139-11140.

35. Israelachvili, J.N.; Drummond, C. On the conformational state of molecules in molecularly thin shearing films. Proc. Natl. Acad. Sci. USA 2015, 112, E4973, doi:10.1073/pnas.1510758112.

36. Rosenhek-Goldian, I.; Kampf, N.; Yeredor, A.; Klein, J. Reply to Jee et al. and Israelachvili and Drummond: Lubricant films do not fluidize in intermittent stick-slip friction. Proc. Natl. Acad. Sci. USA 2015, 112, E4974, doi:10.1073/pnas.1511648112.

37. Werzer, O.; Cranston, E.D.; Warr, G.G.; Atkin, R.; Rutland, M.W. Ionic liquid nanotribology: Mica-silica interactions in ethylammonium nitrate. Phys. Chem. Chem. Phys. 2012, 14, 5147-5152.

38. Elbourne, A.; Sweeney, J.; Webber, G.B.; Wanless, E.J.; Warr, G.G.; Rutland, M.W.; Atkin, R. Adsorbed and near-surface structure of ionic liquids determines nanoscale friction. Chem. Commun. 2013, 49, 6797-6799.

39. Gee, M.L.; McGuiggan, P.M.; Israelachvili, J.N.; Homola, A.M. Liquid to solidlike transitions of molecularly thin films under shear. J. Chem. Phys. 1990, 93, 1895-1906. 
40. Kumacheva, E.; Klein, J. Simple liquids confined to molecularly thin layers. II. Shear and frictional behavior of solidified films. J. Chem. Phys. 1998, 108, 7010-7022.

41. Espinosa-Marzal, R.M.; Arcifa, A.; Rossi, A.; Spencer, N.D. Microslips to "Avalanches" in Confined, Molecular Layers of Ionic Liquids. J. Phys. Chem. Lett. 2014, 5, 179-184.

42. Smith, A.M.; Lee, A.A.; Perkin, S. Switching the Structural Force in Ionic Liquid-Solvent Mixtures by Varying Composition. Phys. Rev. Lett. 2017, 118, doi:10.1103/PhysRevLett.118.096002.

43. Hayes, R.; Warr, G.G.; Atkin, R. At the interface: Solvation and designing ionic liquids. Phys. Chem. Chem. Phys. 2010, 12, 1709-1723.

44. Sweeney, J.; Webber, G.B.; Rutland, M.W.; Atkin, R. Effect of ion structure on nanoscale friction in protic ionic liquids. Phys. Chem. Chem. Phys. 2014, 16, 16651-16658.

45. Federici Canova, F.; Matsubara, H.; Mizukami, M.; Kurihara, K.; Shluger, A.L. Shear dynamics of nanoconfined ionic liquids. Phys. Chem. Chem. Phys. 2014, 16, 8247-8256.

46. Hoth, J.; Hausen, F.; Müser, M.H.; Bennewitz, R. Force microscopy of layering and friction in an ionic liquid. J. Phys. Condens. Matter 2014, 26, doi:10.1088/0953-8984/26/28/284110.

47. De Wijn, A.S.; Fasolino, A.; Filippov, A.E.; Urbakh, M. Effects of molecule anchoring and dispersion on nanoscopic friction under electrochemical control. J. Phys. Condens. Matter 2016, 28, doi:10.1088/0953-8984/28/10/105001.

48. Kramer, G.; Hausen, F.; Bennewitz, R. Dynamic shear force microscopy of confined liquids at a gold electrode. Faraday Discuss. 2017, 199, 299-309.

49. Fajardo, O.Y.; Bresme, F.; Kornyshev, A.A.; Urbakh, M. Water in Ionic Liquid Lubricants: Friend and Foe. ACS Nano 2017, 11, 6825-6831.

50. Espinosa-Marzal, R.M.; Arcifa, A.; Rossi, A.; Spencer, N.D. Ionic Liquids Confined in Hydrophilic Nanocontacts: Structure and Lubricity in the Presence of Water. J. Phys. Chem. C 2014, 118, 6491-6503.

51. Cheng, H.W.; Stock, P.; Moeremans, B.; Baimpos, T.; Banquy, X.; Renner, F.U.; Valtiner, M. Characterizing the Influence of Water on Charging and Layering at Electrified Ionic-Liquid/Solid Interfaces. Adv. Mater. Interfaces 2015, 2, doi:10.1002/admi.201500159.

52. Thomaz, J.E.; Lawler, C.M.; Fayer, M.D. The Influence of Water on the Alkyl Region Structure in Variable Chain Length Imidazolium-Based Ionic Liquid/Water Mixtures. J. Phys. Chem. B 2016, 120, 10350-10357.

53. Shah, F.U.; Glavatskih, S.; Antzutkin, O.N. Boron in Tribology: From Borates to Ionic Liquids. Tribol. Lett. 2013, 51, 281-301.

54. Dong, R.; Wen, P.; Zhang, S.; Zhang, C.; Sun, W.; Fan, M.; Yang, D.; Zhou, F.; Liu, W. The synthesis and tribological properties of dicarboxylic acid ionic liquids. Tribol. Int. 2017, 114, 132-140.

55. Sweeney, J.; Webber, G.B.; Atkin, R. Near surface properties of mixtures of propylammonium nitrate with n-alkanols 2. Nanotribology and fluid dynamics. Phys. Chem. Chem. Phys. 2015, 17, 26629-26637.

56. Li, H.; Somers, A.E.; Howlett, P.C.; Rutland, M.W.; Forsyth, M.; Atkin, R. Addition of low concentrations of an ionic liquid to a base oil reduces friction over multiple length scales: A combined nano- and macrotribology investigation. Phys. Chem. Chem. Phys. 2016, 18, 6541-6547.

57. Smith, A.M.; Perkin, S. Influence of Lithium Solutes on Double-Layer Structure of Ionic Liquids. J. Phys. Chem. Lett. 2015, 6, 4857-4861.

58. Hjalmarsson, N.; Atkin, R.; Rutland, M.W. Effect of Lithium Ions on Rheology and Interfacial Forces in Ethylammonium Nitrate and Ethanolammonium Nitrate. J. Phys. Chem. C 2016, 120, 26960-26967.

59. Fajardo, O.Y.; Bresme, F.; Kornyshev, A.A.; Urbakh, M. Electrotunable Lubricity with Ionic Liquid Nanoscale Films. Sci. Rep. 2015, 5, doi:10.1038/srep07698.

60. Fajardo, O.Y.; Bresme, F.; Kornyshev, A.A.; Urbakh, M. Electrotunable Friction with Ionic Liquid Lubricants: How Important Is the Molecular Structure of the Ions? J. Phys. Chem. Lett. 2015, 6, 3998-4004.

61. Capozza, R.; Benassi, A.; Vanossi, A.; Tosatti, E. Electrical charging effects on the sliding friction of a model nano-confined ionic liquid. J. Chem. Phys. 2015, 143, 144703, doi:10.1063/1.4933010.

62. Sweeney, J.; Hausen, F.; Hayes, R.; Webber, G.B.; Endres, F.; Rutland, M.W.; Bennewitz, R.; Atkin, R. Control of Nanoscale Friction on Gold in an Ionic Liquid by a Potential-Dependent Ionic Lubricant Layer. Phys. Rev. Lett. 2012, 109, 155502, doi:10.1103/PhysRevLett.109.155502.

63. Li, H.; Rutland, M.W.; Atkin, R. Ionic liquid lubrication: influence of ion structure, surface potential and sliding velocity. Phys. Chem. Chem. Phys. 2013, 15, 14616-14623. 
64. Li, H.; Atkin, R.; Page, A.J. Combined friction force microscopy and quantum chemical investigation of the tribotronic response at the propylammonium nitrate-graphite interface. Phys. Chem. Chem. Phys. 2015, 17, 16047-16052.

65. Li, H.; Wood, R.J.; Rutland, M.W.; Atkin, R. An ionic liquid lubricant enables superlubricity to be "switched on" in situ using an electrical potential. Chem. Commun. 2014, 50, 4368-4370.

66. Li, H.; Rutland, M.W.; Watanabe, M.; Atkin, R. Boundary layer friction of solvate ionic liquids as a function of potential. Faraday Discuss. 2017, 199, 311-322.

67. Drummond, C. Electric-Flield-Ilnduced Flriction Rleduction and Clontrol. Phys. Rev. Lett. 2012, 109, doi:10.1103/PhysRevLett.109.154302.

68. Kimura, Y.; Nakano, K.; Kato, T.; Morishita, S. Control of friction coefficient by applying electric fields across liquid crystal boundary films. Wear 1994, 175, 143-149.

69. Nakano, S.; Mizukami, M.; Kurihara, K. Effect of confinement on electric field induced orientation of a nematic liquid crystal. Soft Matter 2014, 10, 2110-2115.

70. Cooper, P.K.; Li, H.; Rutland, M.W.; Webber, G.B.; Atkin, R. Tribotronic control of friction in oil-based lubricants with ionic liquid additives. Phys. Chem. Chem. Phys. 2016, 18, 23657-23662.

71. Bordes, R.; Marty, J.D.; Lauth-de Viguerie, N. Room-Temperature Zwitterionic Ionic Liquids. Fr. Ukr. J. Chem. 2016, 4, 85-94.

(C) 2018 by the authors. Licensee MDPI, Basel, Switzerland. This article is an open access article distributed under the terms and conditions of the Creative Commons Attribution (CC BY) license (http:/ / creativecommons.org/licenses/by/4.0/). 